\title{
Conditional Generation Scheme for Entangled Vacuum Evacuated Coherent States by Mixing Two Coherent Beams with a Squeezed Vacuum State
}

\author{
Sun-Hyun Youn* \\ Department of Physics, Chonnam National University, Gwangju 500-757, Korea
}

\begin{abstract}
Conditions to generate high-purity entangled vacuum-evacuated coherent states $\left(|0>| \alpha>^{0}\right.$ $-\left|-\alpha>^{0}\right| 0>$ were studied for two cascade-placed beam splitters, with one squeezed state input and two coherent state inputs whenever a single photon is detected. Controlling the amplitudes and the phases of the beams allows for various amplitudes of the vacuum-evacuated coherent states $\left(\left|\alpha>^{0}=\right| \alpha>-e^{-|\alpha|^{2}} \mid 0>\right)$ up to $\alpha=2.160$ to be manipulated with high-purity.
\end{abstract}

PACS numbers: 42.25.-p, 03.65.-w, 42.50. Lc

Keywords: Nonclassical light, Squeezed state, Entangled coherent state, Photonic state engineering

* email: sunyoun@chonnam.ac.kr, fax: +82-62-530-3369 


\section{INTRODUCTION}

Quantum information science has taken advantage of the basic quantum nature of the world. Superposition and entanglement are the most important properties in quantum information science. Superposition of states makes it possible to build a qubit which is a basic logic element of the quantum computer. A qubit is a state $(\alpha|0>+\beta| 1>)$ different from the classical bit state $\mid 0>$ or $\mid 1>$. The superposed quantum state is relatively easy to generate for small photon numbers, but it's challenging to generate macroscopic superposed states such as that of Schrodinger's cat.

The other quantum nature is entanglement which has no classical analogy. Twin photon generation by parametric amplification is one of the good examples. Entangled states at the single photon level have played huge roles in quantum information science. However, generating a macroscopic entangled state is very difficult. The entangled coherent state is one of the macroscopic entangled states. After Sanders theoretical work on entangled coherent states, several groups studied entangled coherent states by both theoretical and experimental methods [1].

Using a nonlinear Mach-Zehnder interferometer, a pair of coherent states might be transformed into an entangled superposition of coherent states [2]. However, in order to generate an entangled superposition of coherent states, an ultra high Kerr effect should be obtained. A scheme to generate a macroscopic superposition of coherent states using a beam splitter, homodyne measurement, and a very small Kerr nonlinear effect was proposed by H. Jeong [3]. Furthermore, three mode W-type entangled coherent states[4] using a single-photon source and Kerr nonlinearities was suggested [5]. K. Park et. al. proposed schemes to implement the superposition of coherent displacements and squeezings on two beams of light for non-Gaussian entanglement $\left(\left|\alpha>_{1}\right| 0>_{2}+\left|0>_{1}\right| \alpha>_{2}\right)[6]$. With the implementation of a coherent superposition of two distinct quantum operations, the following hybrid entanglement is experimentally demonstrated [7]

$$
\frac{1}{\sqrt{2}}(|0>| \alpha>+|1>|-\alpha>) \text {. }
$$

A macroscopic quantum entangled state is essential for developing quantum information science. One of the simple applications is for measuring the phase precisely. Joo et. al. presented an improved phase estimation scheme employing entangled coherent states and showed that entangled coherent states gave the smallest variance in the phase parameter 
under perfect and lossy conditions [8]. Recently a new scheme was proposed for measuring a phase to a precision significantly better than that attainable by both unentangled classical states and highly-entangled NOON states over a wide range of different losses with a realistic and practical technology [9].

Our work is to demonstrate how to generate entangled coherent states with a squeezed light, two coherent lights, two beam splitters and single photon counters. In our proposed system, we added two coherent beams with cascade-placed beam splitters to the nonclassical state. The two beam splitters and the two coherent beams give us a degree of freedom to control the output in a highly nonclassical manner. We characterize the two outputs from the three input beams with a detection of a single photon. Our system has a great advantage in that it can generate a high-fidelity Fock state [10-12].

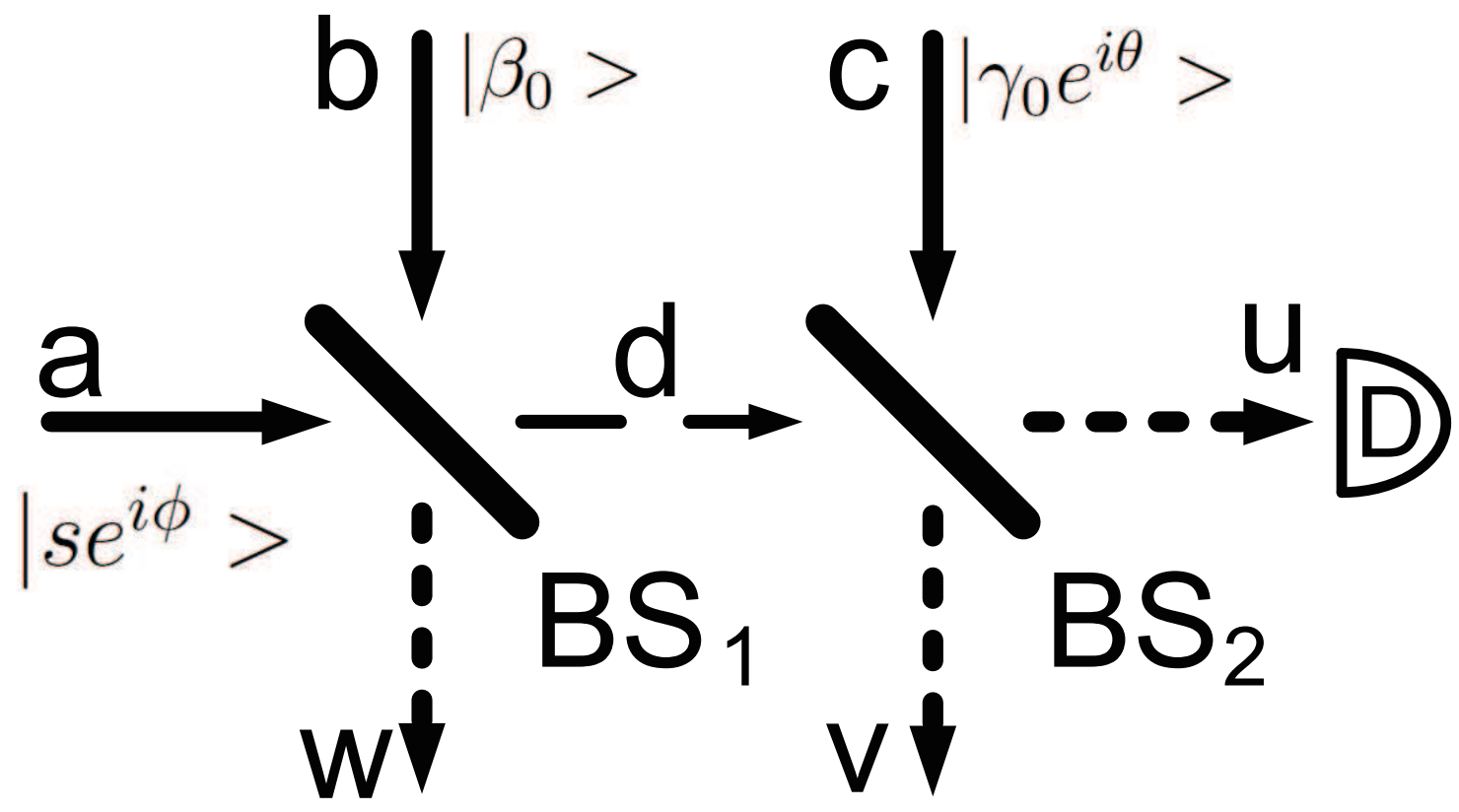

FIG. 1: Schematic diagram of entangled coherent state generation. One squeezed state is in the input mode $a\left(\mid s e^{i \phi}>\right)$, and two coherent states $\left(\left|\beta_{0}>,\right| \gamma_{0} e^{i \theta}>\right)$ are in the input modes $b$ and $c$. BS: Beam Splitter. D: Detector.

The present paper is organized as follows: In Section II, we introduce two-cascade-placed beam splitters with one squeezed state input and two coherent state inputs. We explicitly calculate the probability of the amplitude when a detector at the output port detects a single photon. In section III, we find the analytic condition for which an entangled quantum state 
is generated at the output ports $w$ and $v$ when a single photon is detected at the output port $u$. In Section IV, we numerically find the conditions for an entangled vacuum evacuated coherent state and characterize the entangled state. In Section V, we summarize the main results and discuss the experimental implementation.

\section{TWO CASCADE PLACED BEAM SPLITTERS}

Let a squeezed vacuum state $\mid \xi>$ be in the input mode $a$, and the two coherent states, $\mid \beta>$ and $\mid \gamma>$, be in the input modes $b$ and $c$, as seen in the experimental set up in Fig. 1. Then, the input states $|\xi>,| \beta>$, and $\mid \gamma>$ can be expressed in the number-state representation [13]:

$$
\left|\xi, \beta, \gamma>=e^{-\frac{1}{2}\left(|\beta|^{2}+|\gamma|^{2}\right)} \sum_{n=0, l=0, m=0} C_{n}(\xi) \frac{\left(\hat{a}^{\dagger}\right)^{n}}{\sqrt{n !}} \frac{\left(\beta \hat{b}^{\dagger}\right)^{l}}{l !} \frac{\left(\gamma \hat{c}^{\dagger}\right)^{m}}{m !}\right| 0>_{a}\left|0>_{b}\right| 0>_{c}
$$

where $C_{n}(\xi)$ is the coefficient of the squeezed vacuum with squeezing parameter, $s e^{i \phi}$, and is zero for all odd values of $n$ and nonzero only for an even value of $n$. The nonzero values of $C_{n}(\xi)$ for even values of $n$ become [13]

$$
C_{n}(\xi)=\frac{\sqrt{n !}}{\sqrt{\cosh s} \frac{n}{2} !}\left(-\frac{1}{2} e^{i \phi} \tanh s\right)^{\frac{n}{2}} .
$$

With the experimental set up of Fig. 1, the three creation operators $\hat{a}^{\dagger}, \hat{b}^{\dagger}, \hat{c}^{\dagger}$ are written in terms of three creation operators $\hat{w}^{\dagger}, \hat{v}^{\dagger}, \hat{u}^{\dagger}$ in output modes $u, v$, and $w$. Using an operator relation [14], we can obtain the relationships between the input modes and the output modes as the following:

$$
\begin{aligned}
& \left(\begin{array}{c}
\hat{a}^{\dagger} \\
\hat{b}^{\dagger}
\end{array}\right)=\left(\begin{array}{cc}
t_{1} e^{-i \phi_{\tau_{1}}} & \sqrt{1-t_{1}^{2}} e^{-i \phi_{\rho_{1}}} \\
-\sqrt{1-t_{1}^{2}} e^{i \phi_{\rho_{1}}} & t_{1} e^{i \phi_{\tau_{1}}}
\end{array}\right)\left(\begin{array}{c}
\hat{d}^{\dagger} \\
\hat{w}^{\dagger}
\end{array}\right), \\
& \left(\begin{array}{c}
\hat{d}^{\dagger} \\
\hat{c}^{\dagger}
\end{array}\right)=\left(\begin{array}{cc}
t_{2} e^{-i \phi_{\tau_{2}}} & \sqrt{1-t_{2}^{2}} e^{-i \phi_{\rho_{2}}} \\
-\sqrt{1-t_{2}^{2}} e^{i \phi_{\rho_{2}}} & t_{2} e^{i \phi_{\tau_{2}}}
\end{array}\right)\left(\begin{array}{c}
\hat{u}^{\dagger} \\
\hat{v}^{\dagger}
\end{array}\right) .
\end{aligned}
$$

Then the three input creation operators $\hat{a}^{\dagger}, \hat{b}^{\dagger}, \hat{c}^{\dagger}$ are written in terms of the three output creation operators $\hat{w}^{\dagger}, \hat{v}^{\dagger}, \hat{u}^{\dagger}$ as follows:

$$
\begin{aligned}
& \hat{a}^{\dagger}=q_{a}^{u} \hat{u}^{\dagger}+q_{a}^{v} \hat{v}^{\dagger}+q_{a}^{w} \hat{w}^{\dagger} \\
& \hat{b}^{\dagger}=q_{b}^{u} \hat{u}^{\dagger}+q_{b}^{v} \hat{v}^{\dagger}+q_{b}^{w} \hat{w}^{\dagger} \\
& \hat{c}^{\dagger}=q_{c}^{u} \hat{u}^{\dagger}+q_{c}^{v} \hat{v}^{\dagger},
\end{aligned}
$$


where $q_{\mu}^{\nu}(\mu=a, b, c, \nu=u, v, w)$ represents the relations between the operators in the input modes $\left(\hat{a}^{\dagger}, \hat{b}^{\dagger}, \hat{c}^{\dagger}\right)$ and those in the output modes $\left(\hat{u}^{\dagger}, \hat{v}^{\dagger}, \hat{w}^{\dagger}\right)$ as follows [11]:

$$
\begin{aligned}
\left\{q_{a}^{u}, q_{a}^{v}, q_{a}^{w}\right\} & =\left\{e^{-i\left(\phi_{\tau_{1}}+\phi_{\tau_{2}}\right)} t_{1} t_{2}, e^{-i\left(\phi_{\rho_{2}}+\phi_{\tau_{1}}\right)} t_{1} \sqrt{1-t_{2}^{2}}, e^{-i \phi_{\rho_{1}}} \sqrt{1-t_{1}^{2}}\right\}, \\
\left\{q_{b}^{u}, q_{b}^{v}, q_{b}^{w}\right\} & =\left\{e^{i \phi_{\rho_{1}}} \sqrt{1-t_{1}^{2}} e^{-i \phi_{\tau_{2}}} t_{2},-e^{i \phi_{\rho_{1}}} \sqrt{1-t_{1}^{2}} e^{-i \phi_{\rho_{2}}} \sqrt{1-t_{2}^{2}}, e^{i \phi_{\tau_{1}}} t_{1}\right\}, \\
\left\{q_{c}^{u}, q_{c}^{v}\right\} & =\left\{-e^{i \phi_{\rho_{2}}} \sqrt{1-t_{2}^{2}}, e^{i \phi_{\tau_{2}}} t_{2}\right\} .
\end{aligned}
$$

Then, the input states in Eq. 2 can be written as number-state representations of the output modes $(u, v, w)$ as follows [10]:

$$
\begin{aligned}
\mid \xi, \beta, \gamma> & =e^{-\frac{1}{2}\left(|\beta|^{2}+|\gamma|^{2}\right)} \sum_{n^{\prime}=0, l^{\prime}=0, m^{\prime}=0} C_{n}(\xi) \beta^{l} \gamma^{m} \frac{\sqrt{n !}}{n_{u} ! n_{v} ! n_{w} !} \frac{1}{l_{u} ! l_{v} ! l_{w} !} \frac{1}{m_{u} ! m_{v} !} \\
& \times\left(q_{a}^{u}\right)^{n_{u}}\left(q_{a}^{v}\right)^{n_{v}}\left(q_{a}^{w}\right)^{n_{w}}\left(q_{b}^{u}\right)^{l_{u}}\left(q_{b}^{v}\right)^{l_{v}}\left(q_{b}^{w}\right)^{l_{w}}\left(q_{c}^{u}\right)^{m_{u}}\left(q_{c}^{v}\right)^{m_{v}} \\
& \times\left(\hat{u}^{\dagger}\right)^{n_{u}+l_{u}+m_{u}}\left(\hat{v}^{\dagger}\right)^{n_{v}+l_{v}+m_{v}}\left(\hat{w}^{\dagger}\right)^{n_{w}+l_{w}}\left|0>_{u}\right| 0>_{v} \mid 0>_{w},
\end{aligned}
$$

where the $p^{\prime}\left(p^{\prime}=n^{\prime}, l^{\prime}, m^{\prime}\right)$ summation indicates all summations for non-negative numbers $p_{u}, p_{v}$, and $p_{w}$ such that $p_{u}+p_{v}+p_{w}=p(p=n, l, m)$. When the detector in the $u$ mode detects a single photon, the state in Eq. 8 can be written as follows:

$$
\begin{aligned}
\left(\mid 1>_{u}\right)^{\dagger} \mid \xi, \beta, \gamma> & =e^{-\frac{1}{2}\left(|\beta|^{2}+|\gamma|^{2}\right)} \sum_{n_{u}+l_{u}+m_{u}=1} C_{n}(\xi) \beta^{l} \gamma^{m} \frac{\sqrt{n !}}{n_{u} ! n_{v} ! n_{w} !} \frac{1}{l_{u} ! l_{v} ! l_{w} !} \frac{1}{m_{u} ! m_{v} !} \\
& \times\left(q_{a}^{u}\right)^{n_{u}}\left(q_{a}^{v}\right)^{n_{v}}\left(q_{a}^{w}\right)^{n_{w}}\left(q_{b}^{u}\right)^{l_{u}}\left(q_{b}^{v}\right)^{l_{v}}\left(q_{b}^{w}\right)^{l_{w}}\left(q_{c}^{u}\right)^{m_{u}}\left(q_{c}^{v}\right)^{m_{v}} \\
& \times\left(\hat{v}^{\dagger}\right)^{n_{v}+l_{v}+m_{v}}\left(\hat{w}^{\dagger}\right)^{n_{w}+l_{w}}\left|0>_{v}\right| 0>_{w}, \\
& =\sum_{N_{v}=0, N_{w}=0} C\left(N_{u}=1, N_{v}, N_{w}\right)\left|N_{v}>\right| N_{w}>,
\end{aligned}
$$

where we used new variables $N_{u}=n_{u}+l_{u}+m_{u}, N_{v}=n_{v}+l_{v}+m_{v}$, and $N_{w}=n_{w}+l_{w}$, and $\left|C\left(N_{u}=1, N_{v}, N_{w}\right)\right|^{2}$ is the probability of finding $N_{v}, N_{w}$ photons in the output modes $v, w$ when a detector in the $u$ mode detects a single photon.

\section{ANALYTIC CONDITIONS TO GENERATE AN ENTANGLED STATE}

In order to find the generating condition for the entangled coherent state, we investigate the coefficient $C\left(N_{u}=1, N_{v}=n, N_{w}=m\right)$ in detail. Let us find the state entangled coherent state by first finding the analytic solution for generating the state $\left|0>_{v}\right| \psi>_{w}$. If 
we collected the coefficients for $N_{u}=n_{u}+l_{u}+m_{u}=1$ and $N_{v}=n_{v}+l_{v}+m_{v}=0$ from the Eq. 9 with $n_{v}=l_{v}=m_{v}=0$ and $n_{u}+l_{u}+m_{u}=1$, then Eq. 9 becomes

$$
\begin{aligned}
\left|0>_{v}\right| \psi>_{w} & =e^{-\frac{1}{2}\left(|\beta|^{2}+|\gamma|^{2}\right)} \sum_{N_{w}=l_{w}+n_{w}} C_{n_{u}+n_{w}}(\xi) \beta^{l_{u}+l_{w}} \gamma^{m_{u}} \frac{\sqrt{\left(n_{u}+n_{w}\right) !}}{n_{u} ! n_{w} !} \frac{1}{l_{u} ! l_{w} !} \frac{1}{m_{u} !} \\
& \times\left(q_{a}^{u}\right)^{n_{u}}\left(q_{a}^{w}\right)^{n_{w}}\left(q_{b}^{u}\right)^{l_{u}}\left(q_{b}^{w}\right)^{l_{w}}\left(q_{c}^{u}\right)^{m_{u}}\left(\hat{w}^{\dagger}\right)^{n_{w}+l_{w}}\left|0>_{v}\right| 0>_{w} .
\end{aligned}
$$

$\left(n_{u}, l_{u}, m_{u}\right)$ set has three cases $(1,0,0),(0,1,0),(0,0,1)$ for $n_{u}+l_{u}+m_{u}=1$, and so $\mid \psi>_{w}$ in Eq. 11 can be written

$$
\begin{aligned}
\mid \psi>_{w} & =e^{-\frac{1}{2}\left(|\beta|^{2}+|\gamma|^{2}\right)} \sum_{N_{w}=l_{w}+n_{w}} \beta^{l_{w}}\left(q_{a}^{w}\right)^{n_{w}}\left(q_{b}^{w}\right)^{l_{w}} \frac{1}{l_{w} ! n_{w} !} \\
& \times\left[C_{1+n_{w}}(\xi) q_{a}^{u} \sqrt{\left(1+n_{w}\right) !}+C_{n_{w}} \sqrt{n_{w} !}\left(\beta q_{b}^{u}+\gamma q_{c}^{u}\right)\right]\left(\hat{w}^{\dagger}\right)^{n_{w}+l_{w}} \mid 0>_{w} .
\end{aligned}
$$

If we set the amplitude of the two modes $(\beta, \gamma)$ and the transmittance of two beam splitters such that

$$
\beta q_{b}^{u}+\gamma q_{c}^{u}=0
$$

using the Eq. 7, the transmittance $t_{2}$ can be found which satisfy Eq. 13 for a given $\beta, \gamma, t_{1}$

$$
t_{2}=\frac{\gamma}{\sqrt{\beta^{2}+\gamma^{2}-\beta^{2} t_{1}}}
$$

Then $\mid \psi>_{w}$ can be written

$$
\begin{aligned}
\mid \psi>_{w} & =e^{-\frac{1}{2}\left(|\beta|^{2}+|\gamma|^{2}\right)} \sum_{N_{w}=l_{w}+n_{w}} \beta^{l_{w}}\left(q_{a}^{w}\right)^{n_{w}}\left(q_{b}^{w}\right)^{l_{w}} \frac{1}{l_{w} ! n_{w} !} \\
& \times C_{1+n_{w}}(\xi) q_{a}^{u} \sqrt{\left(1+n_{w}\right) !}\left(\hat{w}^{\dagger}\right)^{n_{w}+l_{w}} \mid 0>_{w} .
\end{aligned}
$$

Using $C_{1+n_{w}}(\xi)$ in Eq. 3,

$$
\begin{aligned}
\mid \psi>_{w} & =e^{-\frac{1}{2}\left(|\beta|^{2}+|\gamma|^{2}\right)} \sum_{N_{w}=l_{w}+n_{w}} \beta^{l_{w}}\left(q_{a}^{w}\right)^{n_{w}}\left(q_{b}^{w}\right)^{l_{w}} \frac{1}{l_{w} ! n_{w} !} \\
& \times \frac{\sqrt{\left(1+n_{w}\right) !}}{\sqrt{\cosh s} \frac{1+n_{w}}{2} !}\left(-\frac{1}{2} e^{i \phi} \tanh s\right)^{\frac{1+n_{w}}{2}} q_{a}^{u} \sqrt{\left(1+n_{w}\right) !}\left(\hat{w}^{\dagger}\right)^{n_{w}+l_{w}} \mid 0>_{w},
\end{aligned}
$$

where $n_{w}$ should be odd. Then the coefficient for $N_{w}=l_{w}+n_{w}=0$ is zero, and the probability that $\mid \psi>_{w}$ is in a vacuum state is zero. Therefore, $\mid \psi>_{w}$ can not be a coherent state $(\mid \alpha>)$. With integer $k$ such that $1+n_{w}=2 k$, and $l_{w}=N_{w}-2 k+1$ 


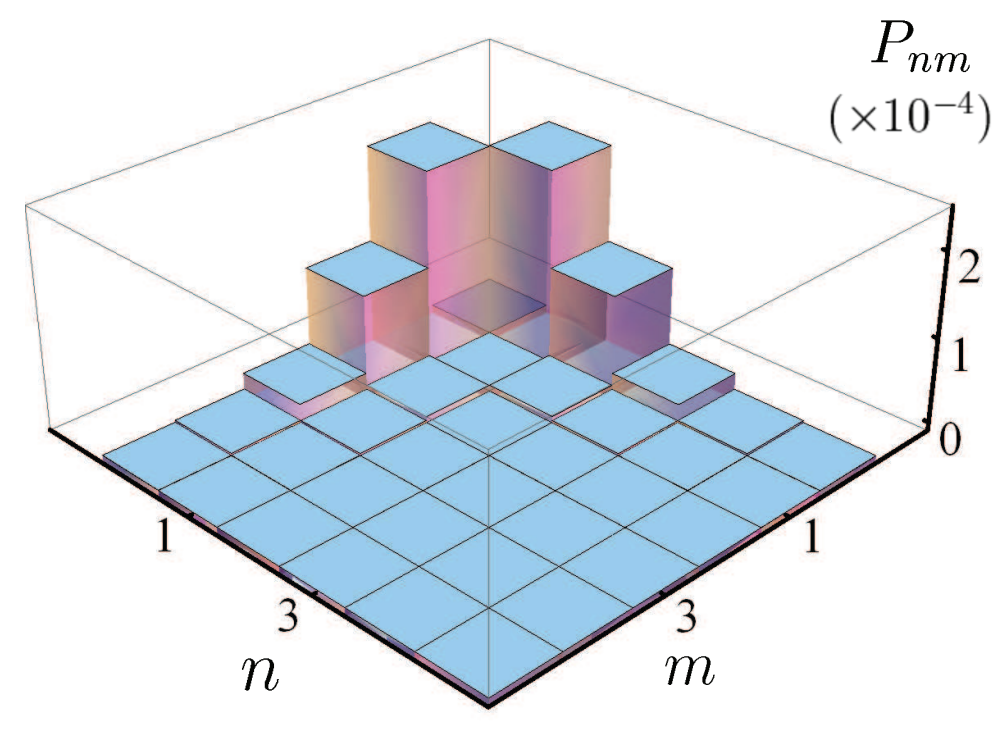

FIG. 2: Probability function $P_{n m}$ of the generated states with $\alpha=\beta=\frac{1}{2}, s=\frac{1}{2}, t_{1}=0.999$ and $t_{2}=0.999$.

$$
\begin{aligned}
\mid \psi>_{w} & =e^{-\frac{1}{2}\left(|\beta|^{2}+|\gamma|^{2}\right)} \frac{2 q_{a}^{u}}{\sqrt{\cosh s}} \sum_{N_{w}=1} \sum_{k=1}^{\frac{N_{w}+1}{2}}\left(\beta q_{b}^{w}\right)^{N_{w}}\left(\frac{q_{a}^{w}}{\beta q_{b}^{w}}\right)^{2 k-1} \\
& \times \frac{1}{\left(N_{w}-2 k+1\right) !(k-1) !}\left(-\frac{1}{2} e^{i \phi} \tanh s\right)^{k}\left(\hat{w}^{\dagger}\right)^{N_{w}} \mid 0>_{w} .
\end{aligned}
$$

Considering the summation over $k$, two terms compete. One of them is $\left|\left(\frac{q_{a}^{w}}{\beta q_{b}^{w}}\right)^{2}\left(-\frac{1}{2} e^{i \phi} \tanh s\right)\right|^{k}$, which decrease as $k$ increases if $\left|\left(\frac{q_{a}^{w}}{\beta q_{b}^{w}}\right)^{2}\left(-\frac{1}{2} e^{i \phi} \tanh s\right)\right|<1$. The other one is $\frac{1}{\left(N_{w}-2 k+1\right) !(k-1) !}$, which increase as $k$ increases. So the summation over $k$ can not turn into a simple analytic form. However, if the following condition satisfied

$$
\left|\left(\frac{q_{a}^{w}}{\beta q_{b}^{w}}\right)^{2}\left(-\frac{1}{2} e^{i \phi} \tanh s\right)\right|<<1,
$$

the dominant term in the $k$ summation is only for $k=1$, and then $\mid \psi>_{w}$ becomes

$$
\begin{aligned}
\mid \psi>_{w} & \sim e^{-\frac{1}{2}\left(|\beta|^{2}+|\gamma|^{2}\right)} \frac{2 q_{a}^{u}}{\sqrt{\cosh s}}\left(\frac{q_{a}^{w}}{\beta q_{b}^{w}}\right)\left(-\frac{1}{2} e^{i \phi} \tanh s\right) \\
& \times \sum_{N_{w}=1}\left(\beta q_{b}^{w}\right)^{N_{w}} \frac{1}{\left(N_{w}-1\right) !}\left(\hat{w}^{\dagger}\right)^{N_{w}} \mid 0>_{w} .
\end{aligned}
$$

The terms in Eq. 19 are not matched to the coherent state, but the forms are similar to the photon added coherent state $\hat{w}^{\dagger} \mid\left(\beta q_{b}^{w}\right)>_{w}$. 
If we set the same amplitudes of two coherent beams $\beta_{0}=\gamma_{0}=\frac{1}{2}, s=\frac{1}{2}$, and $t_{1}=0.999$ , Eq. 14 satisfied with $t_{2}=0.999$. These setups give $\left|\left(\frac{q_{a}^{w}}{\beta q_{b}^{w}}\right)^{2}\left(-\frac{1}{2} e^{i \phi} \tanh s\right)\right|=1.85 \times 10^{-3}$, and then $\mid \psi>_{w}$ may be written as Eq. 19. In Fig. 2, we plot the probability

$$
P_{n m}=\left|C\left(N_{u}=1, N_{v}=n, N_{w}=m\right)\right|^{2} .
$$

calculated from the original Eq. 10. The probability $P_{n m}=0$, if $n \neq 0$ or $m \neq 0$. Although, we calculated the analytic form for the $\left|0>_{v}\right| \psi_{1}>_{w}$ state, the probability distribution $P_{n m}$ in Fig. 2 shows the existence of another state $\left|\psi_{2}>_{v}\right| 0>$. We assumed that the state has a form of the entangled state such as $\left(|0>| \psi_{1}>_{w}+\left|\psi_{2}>_{v}\right| 0>\right)$.

\section{NUMERICAL CONDITIONS TO GENERATE AN ENTANGLED VACUUM EVACUATED COHERENT STATE.}

In order to study the state $\left(|0>| \psi_{1}>_{w}+\left|\psi_{2}>_{v}\right| 0>\right)$ in detail, we release the condition Eq. 18, and we numerically find some entangled quantum state by changing parameters with fixed $s$ and $\gamma_{0}$. Several conditions to generate an entangled quantum state are given in Table I. To generate the state $\mid \Psi_{1}>$, we set the amplitudes of two coherent beams $\beta_{0}=0.813, \gamma_{0}=\frac{1}{2}$, and the squeezing parameter $s=\frac{1}{2}$, and two transmittance of the beam splitters are $t_{1}=0.829$, and $t_{2}=0.740$. With these setups, if we detect a single photon at the $u$ mode, then we are sure that an entangled quantum state $\left|\Psi_{1}\right\rangle$ is generated at the $v$ and $w$ modes. We define the purity $(P u)$

$$
P u=\frac{\sum_{n=0} P_{n 0}+\sum_{m=0} P_{0 m}}{\sum_{n=0, m=0} P_{n m}} .
$$

Then the $\left|\Psi_{1}\right\rangle$ in Table II has $P u=99.8 \%$. The purity $P u=99.8 \%$ means that the generated state is $99.8 \%$ described by $\left(|0>| \psi_{1}>_{w}+\left|\psi_{2}>_{v}\right| 0>\right)$, and others can be

represented $\left(|n>| \psi_{1}>_{w}+\left|\psi_{2}>_{v}\right| m>\right)$, for nonzero $n, m$. In Fig. 3, we plotted the probability $P_{n m}$ for $\mid \Psi_{1}>$ in Table I. We can see that $P_{n m}$ is almost zero if one of the $(n, m)$ is not zero.

The probability $(\mathrm{Pr})$ to make such event is as follows:

$$
\operatorname{Pr}=\sum_{n=0, m=0} P_{n m}
$$

then the $\operatorname{Pr}$ for the $\mid \Psi_{1}>$ in Table $\Pi$ is 0.050 . 


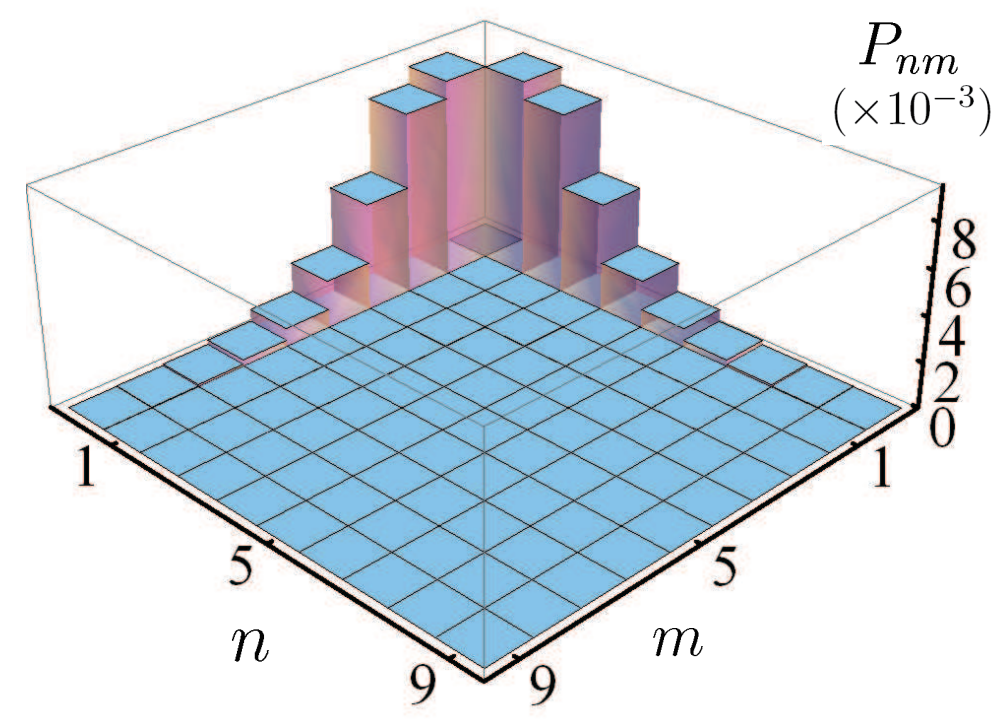

FIG. 3: Probability function $P_{n m}$ of the generated states with $\beta=0.81$.

In order to find the characteristics of the probability amplitude in detail, we put the state $\mid \Psi_{1}>$ as follows:

$$
\mid \Psi_{1}>=f\left(\left|0>_{v}\right| \alpha>_{w}^{0}-\left|-\alpha>_{v}^{0}\right| 0>_{w}\right),
$$

where $\mid \alpha>^{0}\left(\equiv\left|\alpha>-e^{-|\alpha|^{2}}\right| 0>\right)$ is the vacuum evacuated coherent state. We determine the amplitude $\alpha$ and the scale factor $f$ such that minimizes the error functions $E r$ as follows:

$$
E r=\sum_{n=1}^{9}\left(|C(1, n, 0)+f \times c o(-\alpha, n)|^{2}+|C(1,0, n)-f \times c o(\alpha, n)|^{2}\right),
$$

where $\operatorname{co}(\alpha, n)$ is the coefficient such that the state is in the number state $|n\rangle$ for the coherent state with amplitude $\alpha$

$$
\operatorname{co}(\alpha, n)=e^{-|\alpha|^{2}} \alpha^{n} / \sqrt{n !}
$$

Note that, Eq. 24 does not count the $\operatorname{co}(\alpha, 0)$ and the summation is limited at the photon number 9 .

In Fig. 4, we plotted the coefficient $C(1, n, 0)$ defined by Eq. 10 and $(-f \times c o(-\alpha, n))$. The bar chart represents $C(1, n, 0)$ and the joined dots represent $0.174 \times c o(-1.350, n)$. We also plotted the coefficient $C(1,0, n)$ and $f \times c o(\alpha, n)$ in Fig. 5. The joined dots in Fig. 5 were calculated from the coefficient $0.174 \times c o(1.350, n)$. The total error sum is about 
$1.6 \times 10^{-5}$. If we represents the state $\mid \alpha>_{w}^{0}$ as a in number state, the coefficient is the same as that of the coherent state only excepting only the coefficient of the vacuum state.

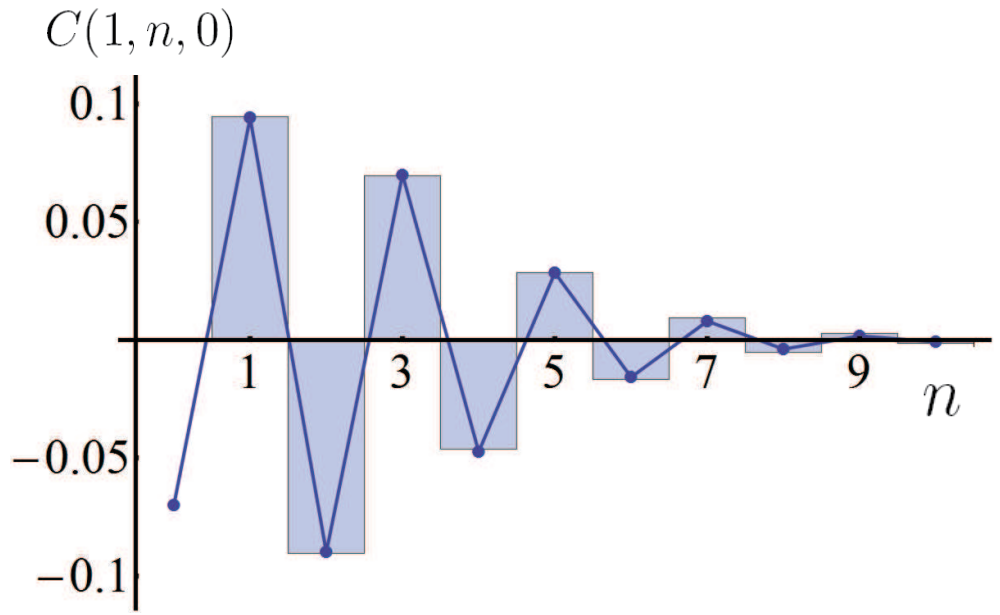

FIG. 4: The bar chart represents the coefficient $C(1, n, 0)$ and the jointed dots represent $(-0.174 \times$ $\operatorname{co}(-1.350, n))$.

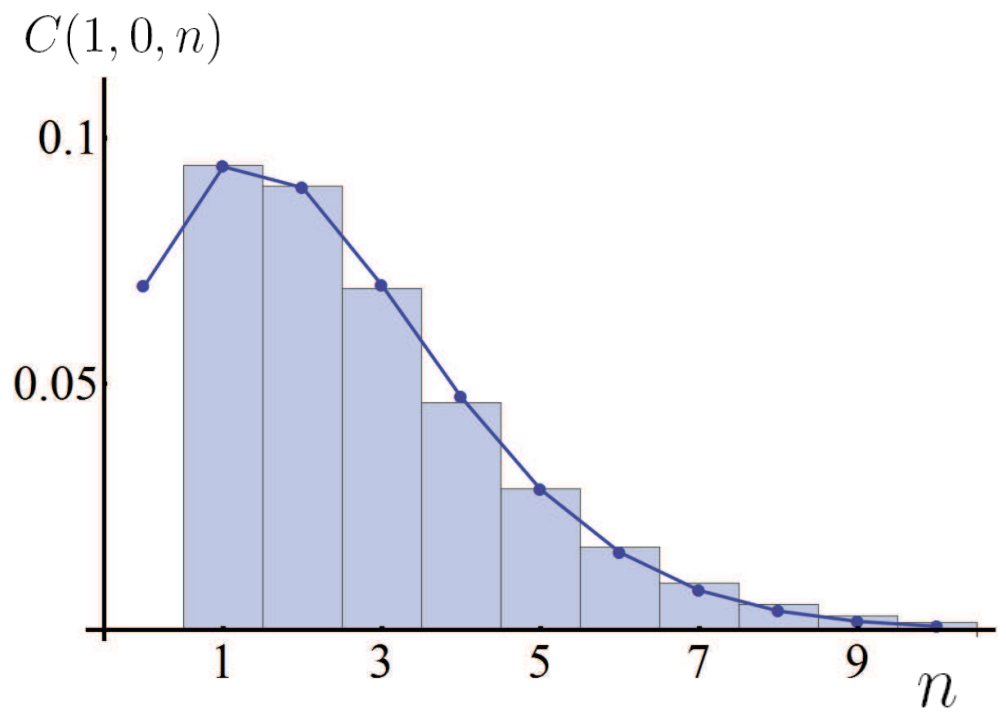

FIG. 5: The bar chart represents the coefficient $C(1,0, n)$ and the jointed dots represent $(0.174 \times$ $c o(1.350, n))$.

The probability amplitude $P_{n m}$ is sensitive to the phase relation among three input beams. In addition to the phase relations among three beams, the phase shift in Eqs. 445 at two beam splitters should be counted. The total phase factors are linear functions of the relative 
phases among the three input beams and the phase shifts at two beam splitters [10], so if we scan the relative phases $\theta$ and $\phi$, we can obtain the phase-dependent probability amplitude $P_{n m}$. For simple notation, we set all the phase shifts at the beam splitters to 0 .

If we changed the phase $\phi$ from $\pi$ to 0 , the probability amplitude $P_{n m}$ is not an entangled vacuum evacuated coherent state any more. If the squeezed sate is divided by a beam splitter, the entanglement between the two outputs is very sensitive to the phase shifts [15]. In actual experiments, it's difficult to control the phase shift of the beam splitter. However, in our scheme, the total phase shifts including the phase shifts at the beam splitter can be scanned by changing the phase differences among the three input beams. The entangled vacuum evacuated coherent state can be obtained using only the phase differences $\phi=\pi$ and $\theta=\pi$.

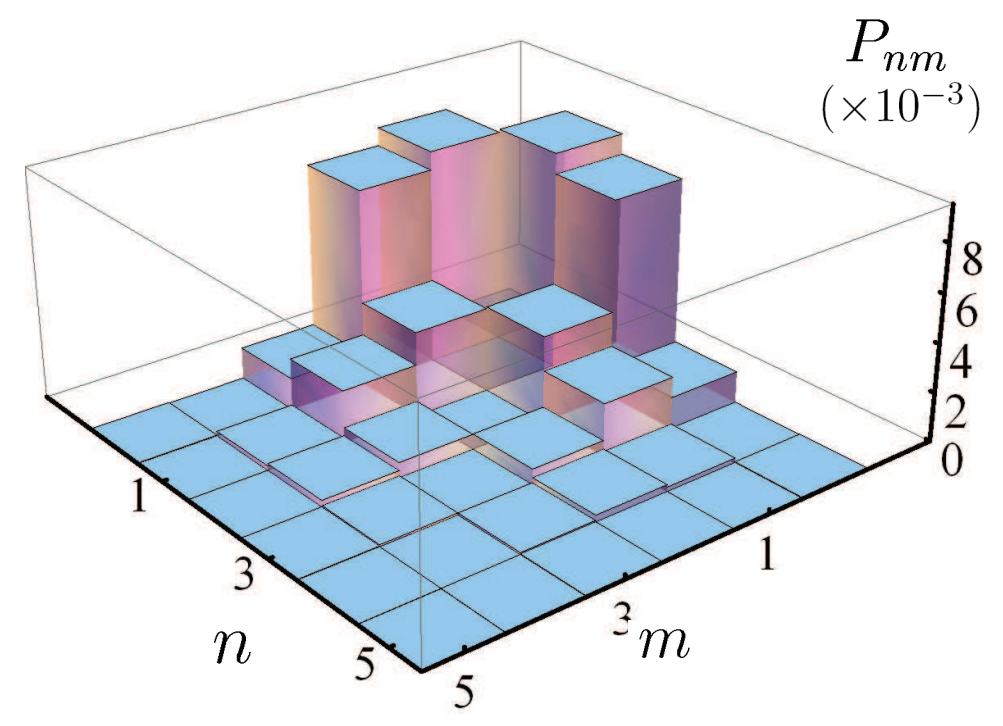

FIG. 6: Probability function $P_{n m}$ of the generated states with $\beta=0.81, s=\frac{1}{2}, \phi=0$.

We showed several conditions to generate an entangled vacuum evacuated coherent state for bigger amplitudes in the Table \. With the input amplitudes $\gamma_{0}=\frac{1}{2}, s=\frac{1}{2}$, we can obtain the amplitude of the entangled vacuum evacuated coherent state amplitudes of $\alpha=1.591$ and $\alpha=1.819$. If we add the amplitude of the squeezed vacuum $s=3 / 4$, the amplitudes of the entangled vacuum evacuated coherent state become $\alpha=1.990$ and $\alpha=2.160$. We plotted the probability amplitude $P_{n m}$ for $\mid \Psi_{4}>=\left(|0>| 1.990>^{0}-\left|-1.990>^{0}\right| 0>\right)$ in 
TABLE I: Conditions for generating the vacuum evacuated entangled coherent state. $(\phi=\pi, \theta=$ $\pi)$

\begin{tabular}{c||c|c|c|c|c|c|c|c|c|r}
\hline state & $s$ & $\beta_{0}$ & $\gamma_{0}$ & $t_{1}$ & $t_{2}$ & $\operatorname{Pr}$ & $\operatorname{Pu}(\%)$ & $\alpha$ & $f$ & $\operatorname{Er}\left(\times 10^{-5}\right)$ \\
\hline $\mid \Psi_{1}>$ & $\frac{1}{2}$ & 0.813 & $\frac{1}{2}$ & 0.829 & 0.740 & 0.050 & 99.8 & 1.350 & 0.174 & 0.16 \\
\hline$\Psi_{2}>$ & $\frac{1}{2}$ & 1.040 & $\frac{1}{2}$ & 0.780 & 0.609 & 0.041 & 99.3 & 1.591 & 0.148 & 0.33 \\
\hline $\mid \Psi_{3}>$ & $\frac{1}{2}$ & 1.292 & $\frac{1}{2}$ & 0.744 & 0.502 & 0.029 & 97.1 & 1.819 & 0.122 & 4.25 \\
\hline$\Psi_{4}>$ & $\frac{3}{4}$ & 1.302 & $\frac{1}{2}$ & 0.729 & 0.491 & 0.059 & 97.1 & 1.990 & 0.171 & 24.8 \\
\hline$\Psi_{5}>$ & $\frac{3}{4}$ & 1.498 & $\frac{1}{2}$ & 0.710 & 0.428 & 0.046 & 96.2 & 2.160 & 0.150 & 18.2 \\
\hline \hline
\end{tabular}

\section{Fig[7.}

The probability $\operatorname{Pr}$ to generate $\left|\Psi_{1}\right\rangle\left|\Psi_{5}\right\rangle$ is around 5\%, and the purity $P u$, is greater than $96 \%$. The err sum $E r$ is increased as $\alpha$ is increased. The main reason is caused by our calculation limit. In Fig. [7, the probability amplitude $P_{09}$ and $P_{90}$ are not zero. We only calculated the Error sum to the photon number 9 in Eq. 24. The main reason we terminated the photon number at 9 is to make the computational and memory size burdens reasonable. Although we terminated the coefficients at 9, the coefficient is the exact one up to photon number 9 in our calculation.

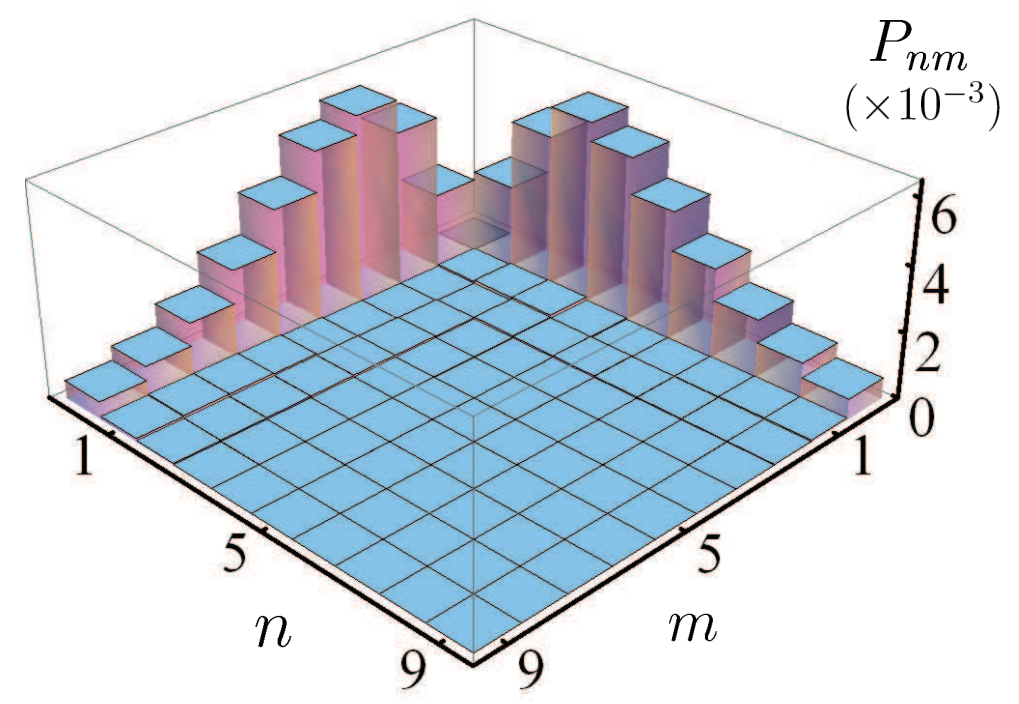

FIG. 7: Probability function $P_{n m}$ of the generated states with $\beta=1.302$. 


\section{DISCUSSION}

The generation of entangled states through the use of a squeezed light source and conditional measurements has been extensively studied both theoretically and experimentally. Our work is to demonstrate how to generate an entangled state with a squeezed light, two coherent lights, two beam splitters and single photon counters. The two beam splitters and the two coherent beams give us a degrees of freedom with which to control the output in a highly nonclassical manner. We characterize the two outputs from the three input beams with the detection of single photon.

Entangled quantum states are indispensable for quantum information science. Furthermore, entangled coherent states have great advantages for applications. In our new scheme to generate an entangled quantum state, we showed the possibility of generating a vacuum evacuated coherent state that is entangled with vacuum state. We find the analytic conditions for which an entangled quantum state is generated at the output port $w$ and $v$ when a single photon is detected at the output port $u$. With the same amplitudes $\beta_{0}=\gamma_{0}=s=\frac{1}{2}$, and $t_{1}=t_{2}=0.999$, an entangled quantum state can be generated.

If we changed the amplitude $\beta_{0}=0.813$, and the two transmittances of the beam splitters are $t_{1}=0.829$, and $t_{2}=0.740$, we can obtain the entangled quantum state $\left(|0>| \psi_{w}>\right.$ $\left.+\left|\psi_{v}>\right| 0>\right)$. The state $\mid \psi_{w}>$ is a vacuum evacuated coherent state $\left(\left|\alpha>^{0}=\right| \alpha>\right.$ $-e^{-|\alpha|^{2}} \mid 0>$ ) with $\alpha=1.350$, and $\left|\psi_{v}>=-\right| \alpha=-1.350>^{0}$. The error sum is less than $10^{-5}$. We can obtain a large amplitude of the entangled vacuum evacuated coherent state up to $\alpha=2.160$ with the amplitude of the squeezed vacuum $s=\frac{3}{4}$. Although the error sum $E r$ is increased as $\alpha$ is increased, the main reason is simply caused by calculation setting limit we set for fast calculation.

With the explicit form, the probability amplitude for an output state is a function of the transmittances of the two beam splitters and the amplitudes and the relative phases of the three input beams. The probabilities are calculated when the a single photon is detected. We have included all of the coefficients of the input beams from zero to 16 of the number representations for the three input states and use the coefficient up to 9 .

Considering applicability to actual experiments [16], if we use the input beam as pulsed light with a repetition rate of $100 \mathrm{MHz}$, then a generation probability $\operatorname{Pr}$ of $10^{-3}$ results in $10^{6}$ signals per second. 
In actual experiments, a entanglement can be reduced as a result of experimental imperfections, such as mode matching and non-unity quantum efficiency. We assumed perfect temporal and spatial mode matching among the three input beams. These assumptions also guaranteed that the spatial and the temporal mode properties of the entangled states generated in our scheme are well defined by the input states and that the modes of the two coherent states and the squeezed vacuum could be precisely controlled by adjusting the pump beam used to produce the squeezed states. We expect high-purity spatial and temporal modes of the entangled state. A large amplitude entangled vacuum evacuated coherent state can be used to study the quantum nature of the world, and it is a key element in quantum technology.

\section{Acknowledgments}

This study was supported by the Basic Science Research Program through the National Research Foundation of Korea (NRF) funded by the Ministry of Education, Science and Technology (NRF-2014R1A1A2055454)

[1] B. C. Sanders, arXiv:1112.1778v1 (2011).

[2] B. C. Sanders, Phys. Rev. A 45, 6811 (1992).

[3] H. Jeong, M. S. Kim, T. C. Ralph, and B. S. Ham, Phys. Rev. A 70, 061801(R) (2004).

[4] N. B. An, Phys. Rev. A 69, 022315 (2004).

[5] H. Jeong, N. B. An, Phys. Rev. A 74, 022104 (2006).

[6] K. Park, P. Marek, and R. Filip, Phy Rev. A 91, 033814 (2005)

[7] H. Jeong, A. Zavatta, M. Kang, S. Lee, L. S. Costanzo, S. Grandi, T. C. Ralph, and M. Bellini, arXiv:1309.6192 v1 (2013).

[8] J. Joo, W. J. Munro, and T. P. Spiller, Phys. Rev. Lett. 107083601 (2011).

[9] P. A. Knott, and J. A. Dunningham, arXiv:1401.3969v1 (2014).

[10] S. H. Youn, J. Korean Phys. Soc. 63, 1559 (2013).

[11] S. H. Youn, J. Korean Phys. Soc. 64, 217 (2014).

[12] S. H. Youn, J. Korean Phys. Soc. 66, 187 (2015). 
[13] R. Loudon, The Quantum Theory of Light (3rd ed.) Oxford University Press, New York, (2000).

[14] R. A. Campos, B. E. A. Saleh, and M. C. Teich, Phys. Rev. A 40, 1371 (1989).

[15] M. S. Kim, W. Son, V. Buzek, and P. L. Knight, Phys. Rev. A 65, 032323 (2002).

[16] R. Dong, A. Tipsmark, A. Laghaout, L. A. Krivitsky, M. Jezek, and U. L. Andersen, J. Opt. Soc. Am. B, 31, 1192 (2014). 